\title{
A stage-scanning laser confocal microscope and protocol for DNA methylation sequencing
}

\author{
Vaithilingam Vaishnavi, Litty Varghese, Baquir Mohammed Jaffar Ali
}

Life Sciences Division, AU-KBC Research Centre, Anna University, Chennai, India.

Email: jaffar@au-kbc.org

Received 25 September 2009; revised 20 October 2009; accepted 25 October 2009.

\begin{abstract}
Recent understanding of the role of epigenetic regulation in health and disease has necessitated the development of newer and efficient methods to map the methylation pattern of target gene. In this article we report construction of a stage-scanning laser confocal microscope (SLCM) and associated protocol that determines the methylation status of target gene. We have adapted restricted Sanger's sequencing where fluorescine labeled primers and dideoxy guanine fraction alone are used for target amplification and termination at cytosine positions. Amplified ssDNA bands are separated in $6 \%$ denaturing PAGE and scanned using SLCM to sequence the positions of methylated cytosines. We demonstrate that our methodology can detect $<100$ femtomoles of DNA, and resolve the position of cytosine within \pm 2 nucleotide. In a calibration run using a designer DNA of 99 bases, our methodology had resolved all the 11 cytosine positions of the DNA. We have further demonstrated the utility of apparatus by mapping methylation status in the Exon-1 region of a gene, E-Cadherin, in the plasma DNA sample of a healthy subject. We believe our approach constitute a low cost alternative to conventional DNA sequencers and can help develop methylation based DNA biomarkers for the diagnosis of disease and in therapeutics.
\end{abstract}

Keywords: Scanning Confocal Microscope; Fluorescence Detection; Methylation; Bisulfite Sequencing; DNA Sequencer

\section{INTRODUCTION}

Cytosine methylation is required for mammalian development [1]. Alterations of CpG island DNA hypermethylation and chromatin modification have been widely documented in human cancers [2,3] and various other diseases [4,5]. Disease-specific DNA methylation alterations present in tissues and plasma samples can serve as diagnostic markers for risk assessment, progression, early detection, treatment prediction and monitoring [6]. Therefore determination of methylation status of target gene is becoming increasingly important in diagnosis and treatment. Most laboratories use high performance liquid chromatography (HPLC) for the detection of 5methylcytosine residues [7]. However, HPLC requires large amount of DNA and significant experimental experience to obtain reproducible results. Both points are impedimental to the large-scale analysis of clinical samples [8]. Bisulfite genomic sequencing provides an efficient method for detecting individual 5-methyl cytosine (5-MeC) residues in genomic DNA [9]. Analysis of cytosine methylation from the bisulfite- treated and PCR-amplified DNA can be determined by either cloning or sequencing individual molecules or by direct PCR sequencing [10]. In cloning approach, it often requires sequencing of 50-100 individual clones to provide an accurate estimate of methylation in the population of molecules. Whereas in sequencing, standard sequencing is performed using DNA sequencers.

In most situations, methylation status of promoter is taken as indicator to decide gene is expressed or repressed. It is determined by methylation-specific PCR (MSP) which detects the presence of methylated DNA in $\mathrm{CpG}$ islands of the promoter [11]. In MSP, primers are designed to bind specifically to bisulfite converted methylated or unmethylated DNA, leading to specific amplification. Advantage of this technique is its high relative sensitivity, ease of design, and low complexity of the reaction, but it is limited to determining the methylation status only in the primer binding region. Therefore, a new strategy is required to know complete methylation status of target gene.

Complete information of methylation status can usually be derived from sequencing the DNA. The current state-of-the-art technology for high-throughput DNA sequencing utilizes capillary array DNA sequencers with laser-induced fluorescence detection [12-15]. In the case of methylation mapping of the genome, it is redundant to 
sequence the bisulfite converted DNA when sequence information of the genome is already known. Any approach which can by-pass the complete sequencing requirement can be more efficient, faster and resource conserving.

In this communication, we report the customized instrument and protocol for fluorescence detection of DNA bands, reporting methylation status in a target gene. An automated stage-scanning laser confocal microscope (SLCM) has been constructed and calibrated using fluorescently labeled oligo DNA of known lengths. Avoiding detail sequencing of the target gene and thus eliminating the use of DNA sequencer has remained a major objective in our endeavor. In addition, our novel approach in the protocol enabled mapping the methylation status of the target gene in detail that can match sequencing of the gene.

\section{METHODS}

\subsection{Sample Preparations for Bisulfite Sequencing}

\subsubsection{DNA Extraction and Sodium Bisulphite Conversion}

Genomic DNA from human blood plasma was isolated from $1 \mathrm{~mL}$ of serum collected from a normal subject using standard phenol/chloroform method and resuspended in $100 \mu \mathrm{L}$ of water [16]. About $1 \mu \mathrm{g}$ of DNA was denatured by $\mathrm{NaOH}$ followed by treatment with sodium bisulfite to convert all unmethylated cytosine to uracil [17]. Bisulfite treated DNA samples were purified using the Wizard DNA clean-up system (Promega Corp. USA), followed by $\mathrm{NaOH}$ treatment and ethanol precipitation. Finally it is resuspended in $20 \mu \mathrm{l}$ of water.

\subsubsection{Amplification of Target DNA}

Following two DNA templates were designed and synthesized for calibration of the instrument. Template A: 5'CGA TCG TAT TCG GCG TTT GTT TTC GTTCGG CGT TTT CGG TTA GTT ATG GGT TTT TGG AGT CGT AGT TTT TCG GCG TTG TTG TTG TTG TTG TAG GTA TTT3' containing $11 \mathrm{CpG}$ spots, and Template B: 5'CGA TAG TAT TAG GCG TTT GTT TTA GTT AGG AGT CTT GGT TAG TTA TGG GTT TTT GGA GTC GTA GTT TTT CGG GTT GTT GTT GTT GTT GTA GGT ATT T3' containing 5 CpG spots, mimicking methylated cytosine positions. Using MethPrimer [18] primer without $\mathrm{CpG}$ dinucleotides and devoid of regions with poly $\mathrm{T} \geq 9$ is designed to amplify ssDNA Templates $\mathrm{A}$ and $\mathrm{B}$, and target in Exon-1 region of E-cadherin. The resulting primer synthesized with Fluorescein label (6-FAM) at 5' is given by: Flu-5' AAA TAC CTA CAA CAA CAA CAA CAA C3' (Ocimum Biosolutions, Hyderabad). Restricted Sanger's sequencing reaction specifically developed for this [19] was performed using SILVER SEQUENCE ${ }^{\mathrm{TM}}$ DNA se- quencing system (Promega Corp. USA) on these templates. In that dideoxy guanine fraction alone is used so as to terminate the amplicons at all cytosine positions which corresponds to methylated cytosines. The optimized thermal cycling condition giving best results were as follows: $95^{\circ} \mathrm{C}$ for $2 \mathrm{~min}, 35$ cycles of $95^{\circ} \mathrm{C}$ for $30 \mathrm{sec}$, $42^{\circ} \mathrm{C}$ for $30 \mathrm{sec}$ and $72^{\circ} \mathrm{C}$ for $60 \mathrm{sec}$.

\subsubsection{Electrophoresis Separation}

Control ssDNA templates of various sizes containing Fluorescine tag at 5 ' end, and the bisulfite PCR products were separated on $6 \%$ denaturing PAGE in $89 \mathrm{mM}$ Tris, $89 \mathrm{mM}$ boric acid, $2 \mathrm{mM}$ ethylenediamine tetra acetic acid (EDTA) at field strength of $10 \mathrm{~V} / \mathrm{cm}$ for various time interval. Before loading the sample into the gel, an aliquot of the PCR mixture prepared above was added to $2 \mu \mathrm{L}$ of glycerol and heated at $95^{\circ} \mathrm{C}$ for $3 \mathrm{~min}$ to denature the amplicons. Concentration of control ssDNA templates were gradually decreased in different lanes to determine lowest possible concentration that can be detected and resolved by the apparatus.

\subsection{Stage-Scanning Laser Confocal Microscope (SLCM)}

The optical layout of the SLCM setup is schematically represented in Figure 1. The apparatus is built around the Nikon TE2000 microscope frame. The sample stage consists of a motorized XY translator having 100 $\mathrm{mm}$ displacement in each axis (Model: DPM Translator with Micro Position Controller, Holmarc, India). A custom built stage plate with $90 \mathrm{~mm}$ by $90 \mathrm{~mm}$ square slot constitute platform for gel scan. The $473 \mathrm{~nm}$ DPSS laser, CW/100 mW (Model: Ceil, Laser Quantum, UK) is used for fluorescence excitation. The laser

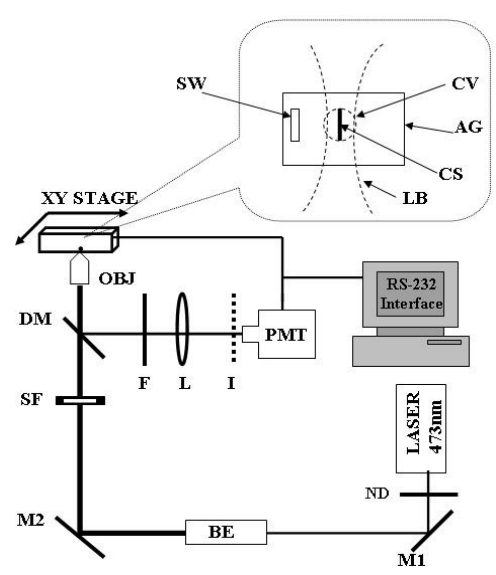

Figure 1. Optical lay-out of stage-scanning laser confocal microscope. OBJ: Objective lens; SF: Spatial filter; DM: Dichroic mirror; M1 and M2: Visible reflecting mirrors; BE: $5 \mathrm{x}$ beam expander; ND: Neutral density filter; F: Emission filter; L: convex lens; I: Iris; PMT: Photomultiplier Tube; LB: laser beam; CV: confocal volume; CS; confocal volume section; SW: Sample well; AG: acrylamide gel. 
beam is expanded to fill the back-focal plane of the 20 $\mathrm{X}$ objective (Nikon). A rectangular slit is introduced as a spatial filter to section the confocal volume to a thin rectangular confocal volume-section. It is aligned parallel to the DNA bands so as to efficiently excite fluorescine tagged to the DNA and minimize the background noise in emission signal. The emission is collected through an iris acting as a confocal spatial filter at left port of the microscope. The photomultiplier tube (Hamamatsu H7468) serves as the detector.

\subsection{Data Acquisition and Control}

XY-Stage and PMT are controlled through RS232 interface. Linear scan is made at a speed of $0.9 \mathrm{~mm} / \mathrm{s}$ and fluorescence signal is acquired by PMT set at $550 \mathrm{~V}, 100$ $\mathrm{ms}$ integration with $0.01 \mathrm{~ms}$ dead time interval. Typical scan of the full length gel is about $80 \mathrm{~mm}$ for $\sim 89 \mathrm{sec}$, giving $\sim 11$ data points per $\mathrm{mm}$ scan. The laser power at the back-focal plane of the objective is $\sim 3.5 \mathrm{~mW}$.

\subsection{Resolution and Sensitivity}

Ability to resolve ssDNA bands is determined by resolving 20 to 43 bases oligo DNA for lower lengths and 73 and 76 bases oligo DNA for longer length. Sensitivity of detection is determined by linear dilution of control ssDNA templates. The optimized scanning and detection protocol given in the above section was followed throughout the measurement of sensitivity \& resolution as well as for mapping the methylation pattern in ssDNA templates and target gene in human genomic DNA.

\section{RESULT AND DISCUSSION}

\subsection{Characterization of SLCM}

\subsubsection{Sensitivity}

Figure 2(a) demonstrates the ability of the apparatus to resolve less than 100 femtomole of fluorescine tagged DNA. The quantity of ssDNA detected is less than typical amount of DNA available in sequencing PCR products in laboratory conditions. Further, perusal of instrument parameters for SLCM suggests that there is more room to improve the fluorescence detection which may bring down the sensitivity level even further.

\subsubsection{Resolution}

Following the same parameters for detection, Figure 2(b) gives the resolution of four short length oligos differing in two bases. In Figure 2(c), resolution of three base differences in longer length oligo is demonstrated. To further demonstrate the ability to resolve multiple bands, ssDNA ladder of eight bands is scanned and given in Figure 2(d). Scrutiny of DNA band separation in Figure 2 suggests that the ability to resolve further is limited by short mobility of bands. In the current configuration, we have practical limitation of $80 \mathrm{~mm}$ scan of the sample which limits the maximum distance over which ssDNA bands can run. In other words, longer scan length will be in a position to resolve the bands to single base resolution. Since methyl sequences can occur at minimum of two bases difference, we have not further explored the ability of the instrument to resolve better.

\subsection{Methylation Mapping in Genomic DNA}

Efficient, unbiased PCR amplification from methylated and unmethylated genomic DNA is essential for any PCR amplification-dependent method designed to detect methylation following bisulphite conversion. Amplicons generated from methylated genomic DNA remains $\mathrm{CpG}$ rich relative to the amplicons from unmethylated genomic DNA, and are often amplified less efficiently. Amplicons from bisulphite-converted genomic DNA typically have homopolymer stretches of 9 or more Ts (As), resulting in poor (or no) amplification. A broadened signal have been observed during electrophoresis for these amplicons due to enzyme "slippage" causing $\mathrm{n}$ +1 and $n-1$ sequences [20]. By selecting amplicons containing no more than 5 consecutive Ts (or As), our PCR was almost always successful. Achieving these two requirements-avoiding homopolymer stretches and designing a primer in a non-CpG region often restricts primer selection to a single possibility for any amplicons within CpG Island.

Following specifically developed restricted Sanger's sequencing protocol [19], two ssDNA templates having known numbers of cytosines were subjected for methyl sequencing. Figure 3(a) gives resolution of all eleven positions present in the ssDNA Template-A, and Figure 3(b) gives all the five positions present the in ssDNA Template-B. In Figure 4(a), methyl sequencing results on the bisulfite treated human genomic DNA at DNA target

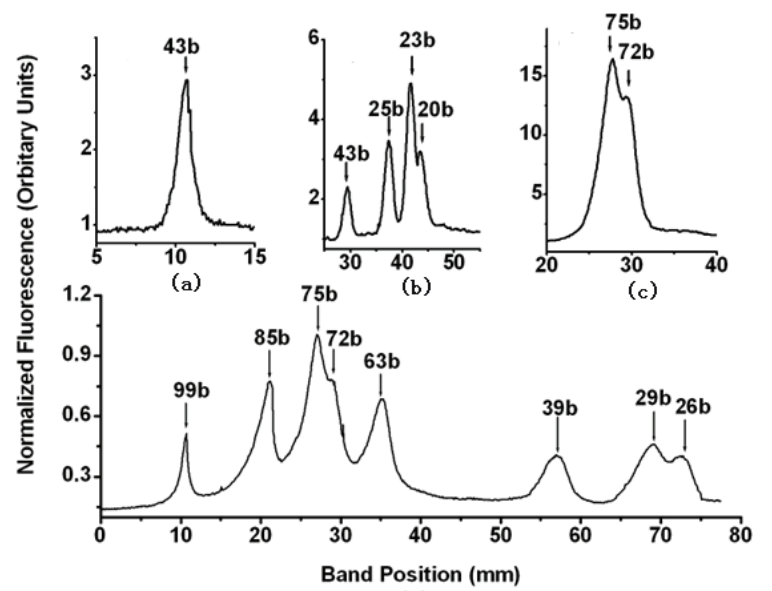

(d)

Figure 2. Demonstrates sensitivity and resolution of SCLM. (a) Detection of 100 femtomoles of ssDNA of 43bases; (b) Resolution of oligos of length 20, 23, 25 and 43 bases; (c) Resolution of oligos of length 72 and 76 bases; (d) Resolution of set of 8 ssDNA Templates length varying from 26 bases to 99 bases. 
on Exon-1 using methylation insensitive primer is given. As a control for resolution of DNA bands, three ssDNA templates of known bases run concurrently on parallel lane is given in Figure 4(b). In Figure 4(c), band position of known control DNA ladder is plotted against the base number. The data is fit to a polynomial to interpolate unknown base information corresponding to position information. For pedagogical reason all possible $\mathrm{CpG}$ positions within 100 bases are plotted (red diamonds). From the precise band position of methyl sequenced DNA in Figure 4(b), and the known corresponding base information, unknown nucleotide sequence is obtained through interpolation of fit in Figure 4(c). It is observed that the target gene had only 30 and 64 base positions from 3' end of the primer found to be methylated. It is noted that only two cytosines are methylated out of 15 available $\mathrm{CpG}$ positions. Ability to retrieve all amplicons in template DNA (Figures 3(a) and 3(b)) suggests that the target sequence in human genomic DNA is partially methylated at the $\mathrm{CpG}$ islands in the promoter region. We emphasis that in prototype resolution of DNA bands in this manner can be software-controlled by inputting the calibration parameters into the system, giving raise to instant reading of target DNA band sequence. Information on methylation sequence position together with quantitative gene expression data can shed more light on the mechanism of epigenetic regulation. More significantly, ability of SLCM to obtain quantitative information on DNA methylation as well as to track its position precisely anywhere in the target sequence can open up many possibilities including development of DNA methylation as biomarkers.

\section{CONCLUSIONS}

It is demonstrated that a customized SLCM apparatus can efficiently resolve ssDNA separation in acrylamide

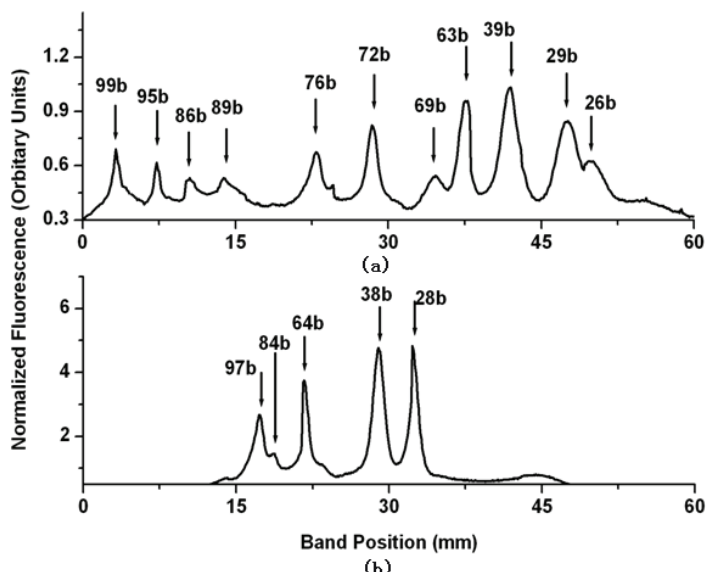

Figure 3. Resolution of methyl sequencing bands in ssDNA Templates. (a) Separation of methylation bands in ssDNA Template-A and; 9b) Separation of bands in ssDNA Template-B.

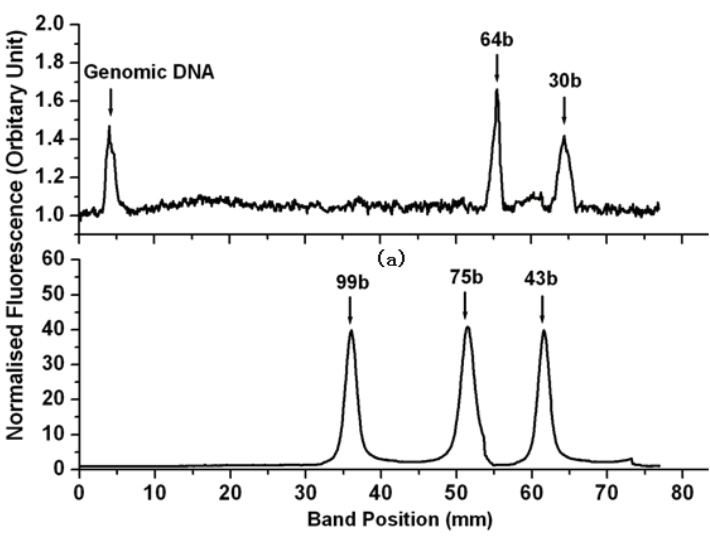

(b)

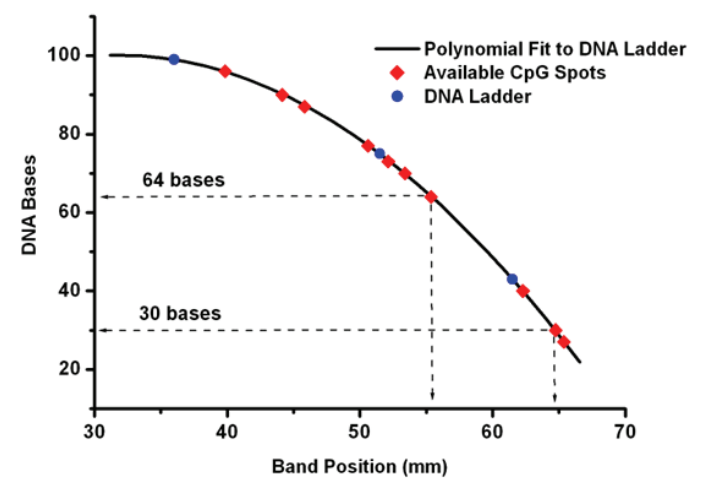

(c)

Figure 4. (a) Methyl sequencing of target gene in human genomic sample using SLCM. Human genomic DNA amplified at the target sequence in Exon-1 region of E-cadhrein, mapped using methyl insensitive in SLCM; (b) Resolution of three known length ssDNA templates runs concurrently with genomic DNA as control; (c) Calibration of DNA band separation distance versus base number. Blue solid circles represents control ladder of known number of bases. Continuous line is fit to the data and red diamonds represents possible $\mathrm{CpG}$ positions within the target region.

gel. We have applied this to map the methylation pattern of target gene. Our SLCM built on low-cost but high sensitive photon detector enabled discrimination of DNA bands of less than 100 femtomoles. Perusal of the parameters of excitation and detection reveal tremendous scope to engineer the sensitivity and resolution in the same configuration. We note that the geometry of fluorescence excitation achieved by using a spatial filter is confocal volume-section instead of volume. It has considerably improved the signal-to-noise. We have also outlined how the current specification can be improved with extension of scan length and modification. Further, $\mathrm{XY}$ scan of gel can enable mapping multiple lanes, thereby sequencing long length of target in a gene or multiple targets in the same genome. We emphasize that normally methylation status away from promoter region or more specifically, away from primer region could not be obtained in any methods except complete sequencing 
of the gene. Striking outcome of the approach presented here is that along with specifically developed methyl sequencing protocol, simple ways to methyl sequence the target gene and access information on the status of methylation away from the promoter region is demonstrated.

\section{ACKNOWLEDGEMENTS}

We thank S. Navaneethakrishnan for his help. This work was supported by Department of Science and Technology, Government of India Grant No. DST/TSG/PT/2006/63.

\section{REFERENCES}

[1] Li, E., Bestor, T.H. and Jaenisch, R. (1992) Targeted mutation of the DNA methyltransferase gene results in embryonic lethality. Cell, 69(6), 915-926.

[2] Jones, P.A. and Baylin, S.B. (2007) The epigenomics of cancer. Cell, 128(4), 683-692.

[3] Toyooka, S. and Shimizu, N. (2004) Models for studying DNA methylation in human cancer: A review of current status. Cancer, 1(1), 37-42.

[4] Post, W.S., et al. (1999) Methylation of the estrogen receptor gene is associated with aging and atherosclerosis in the cardiovascular system. Cardiovascular Research, 43(4), 985-991.

[5] Anselmo, N.P., et al. (2006) Epigenetic alterations in human brain tumors in a brazilian population. Genetics and Molecular Biology, 29(3), 413-422.

[6] Laird, P.W. (2003) The power and the promise of DNA methylation markers. Nature Reviews Cancer, 3(6), 253- 266.

[7] Eick, D., Fritz, H.J. and Doer, W. (1983) Quantitative determination of 5-methylcytosine in DNA by reversephase high-performance liquid chromatography. Analytical Biochemistry, 135(1), 165-171.

[8] Fraga, M.F. and Esteller, M. (2002) DNA methylation: A profile of methods and applications. BioTechniques, 33(3), 632-649.

[9] Jean-Michel, D., Jorg, T., Helene, J. and Ivo, G.G. (2004) De novo quantitative bisulfite sequencing using the pyrosequencing technology. Analytical Biochemistry, 333
(1), 119-127.

[10] Grigg, G.W. and Clark, S.J. (1994) Sequencing 5-methylcytosine residues in genomic DNA. Bioessays, 16(6), 431-436.

[11] Herman, J.G., Graff, J.R., Myohanen, S., Nelkin, B.D. and Baylin, S.B. (1996) Methylation-specific PCR: A novel PCR assay for methylation status of $\mathrm{CpG}$ islands. Proceedings of the National Academy of Sciences, 93(18), 9821-9826.

[12] Stach, D., et al. (2003) Capillary electrophoretic analysis of genomic DNA methylation levels. Nucleic Acids Research, 31(2), e2.

[13] Michaela, W.D.S., Hans-Christian, K., Manfred, W. and Oliverl J.S. (2004) Determination of the DNA methylation level in tumor cells by capillary electrophoresis and laser-induced fluorescence detection. Electrophoresis, 25(6), 839-845.

[14] Clark, S.J., Harrison, J., Paul, C.L. and Frommer, M. (1994) High sensitivity mapping of methylated cytosines. Nucleic Acids Research, 22(15), 2990-2997.

[15] Warnecke, P.M., Stirzaker, C., Melki, J.R., Millar, D.S., Paul, C.L. and Clark, S.J. (1997) Detection and measurement of PCR bias in quantitative methylation analysis of bisulphite-treated DNA. Nucleic Acids Research, 25(21), 4422-4426.

[16] Sambrook, J. and Russell, D.W. (2000) Molecular cloning A laboratory manual. 3rd Edition, Chapter 6.

[17] Marianne, F., et al. (1992) A genomic sequencing protocol that yields a positive display of 5-methylcytosine residues in individual DNA strands. Proceedings of National Academy of Sciences, 89(5), 1827-1832.

[18] http://www.urogene.org/methprimer/index1.html

[19] Vaishnavi, V., Aarthi, R., Smitha, S. and Jaffar Ali, B.M. (2010) A simplified method to determine methylated cytosines in a target gene. IEEE Explore Conference Proceedings, in Press.

[20] Victoria, L.B., Kristina, I.M., Achim, E.K., Kenneth, J.L. G.Z. and John, W.B., (2006) Methylation-dependent fragment separation: Direct detection of DNA methylation by capillary electrophoresis of PCR products from bisulfiteconverted genomic DNA. Analytical Biochemistry, 354(2), 266-273. 